\title{
Evaluation of the Cases Hospitalized in our Burn Unit
}

\author{
Tulay Diken Allahverdi (Corresponding author) \\ Kafkas University, Department of General Surgery, \\ Kars, Turkey \\ Ertugrul Allahverdi \\ Kafkas University, Department of Orthopedics and Traumatology, \\ Kars, Turkey \\ E-mail:drtulaydiken@hotmail.com
}

\begin{abstract}
Aim: A burn is a physiopathological process where damage occurs in the skin and subcutaneous tissues and can affect the entire body depending on its depth and surface area and the reason causing the burn. Burns are most common in children and in the home environment according to a large number of studies. We compared the clinical and demographic features and the treatment protocols of our cases who were diagnosed with a burn in this study.
\end{abstract}

Material and Methods: A total of 52 patients who presented to the Erzincan State Hospital's Burn Unit between July 2008 and June 2010 and were diagnosed with a burn were included in our study. The charts of our cases were retrospectively evaluated. The demographic findings, burn surface area and burn degree determined during the physical examination were recorded from the charts.

\section{Results}

The group of 52 subjects presenting to the burn unit consisted of 33 pediatric and 19 adult patients. The mean age was 18.2 years. There were 17 female and 16 male pediatric patients. The adult patients consisted of 12 females and 7 males. A first-degree burn with an area between $10 \%$ to $15 \%$ of the body surface was found in 12 of our pediatric patients and 8 of our adult patients. All the burns were hot water burns or sunburns. These subjects were prescribed medical treatment for one week and were referred to the outpatient department where the follow-up was performed. The mean duration until recovery was 7 to 10 days. A second-degree burn with a surface area between $25 \%$ and $40 \%$ was found in 15 pediatric patients and 6 adult patients. All these burns had been caused by hot water. Our cases were admitted to the burn unit and treated by dressing the wound. The mean duration until recovery was 4 to 6 weeks. The patients were discharged with full recovery and without any complications. A third-degree burn was found in $6(18.18 \%)$ of our pediatric patients and in $4(21.05 \%)$ of our adult patients. The patients were admitted to the burn unit and dressings alone or with debridement were used for treatment according to the burn healing status.

\section{Conclusion}

Burn patients should be evaluated in detail. Deepening of the burn, infection and fluid loss can be prevented by appropriate intervention in the early period. The appropriate treatment plan should be arranged and the patients who require treatment at a burn center should be referred.

DOI: $10.7176 / \mathrm{JSTR} / 5-12-17$

\section{Introduction}

A burn is a physiopathological process where damage occurs in the skin and subcutaneous tissues and can affect the entire body depending on its depth and surface area and the reason causing the burn. Burns are most common in children, in the home environment, and as a result of hot liquids according to a large number of studies $(1,2,4)$. Around 40 thousand burn patients were admitted to the hospitals in 2013 in the United States of America and the burn was fatal in 3,400 (6).We compared the clinical and demographic features and the treatment protocols of our cases diagnosed with burn in this study.

\section{Material and Method}

A total of 52 patients who presented to the Erzincan State Hospital Burn Unit between July 2008 and June 2010 and were diagnosed with burn were included in this study. Their charts were retrospectively 
investigated. The demographic findings, burn surface area and burn degree as determined in physical examination were recorded from the charts of our cases. The first-degree burns received medical treatment only and were followed-up at the outpatient department. The second-degree burn cases were admitted for treatment. Wound healing was achieved by debridement and dressing in third-degree burns.No complication developed in any of our cases after the treatment and all were discharged with full recovery. The study was approved by the Kafkas University Faculty of Medicine Ethics Committee.

\section{Results}

Of the 52 patients who presented to the burn unit, 33 (63.46\%) were pediatric patients and $19(36.53 \%)$ were adult patients. The mean age was 18.2 years. Of our pediatric patients, 17 were female $(51.51 \%)$ and 16 were male (48.48\%). Of our adult patients, 12 were female $(63.15 \%)$ and 7 were male (36.84\%). A first-degree burn with a surface area between $10 \%$ to $15 \%$ was found in $12(36.36 \%)$ of our pediatric patients and in $8(42.10 \%)$ of our adult patients.(Figure 1). These burns consisted of hot water burns and sunburns. The patients were treated as inpatients for one week and then referred to the outpatient department for follow-up. The mean duration until recovery was 7 to 10 days. A second-degree burn with a surface area of $25 \%$ to $40 \%$ was found in $15(45.45 \%)$ pediatric patients and $6(31.57 \%)$ adult patients.(Figure 2). These burns were hot water burns. Our patients were admitted to the burn unit and their wounds were treated with dressings. The mean duration until recovery was 4 to 6 weeks. Our patients were discharged after healing without any complication. A third-degree burn was found in 6 $(18.18 \%)$ of our pediatric patients and in $4(21.05 \%)$ of our adult patients. (Figure 3).A cautery burn developing during cesarean section was present in one of the four adult patients. The others were due to whey or hot water burns. The patients were admitted to the burn unit and dressings alone or with debridement were used for treatment according to the burn healing status. No complication developed in any of our cases after the treatment. All were discharged with full recovery.
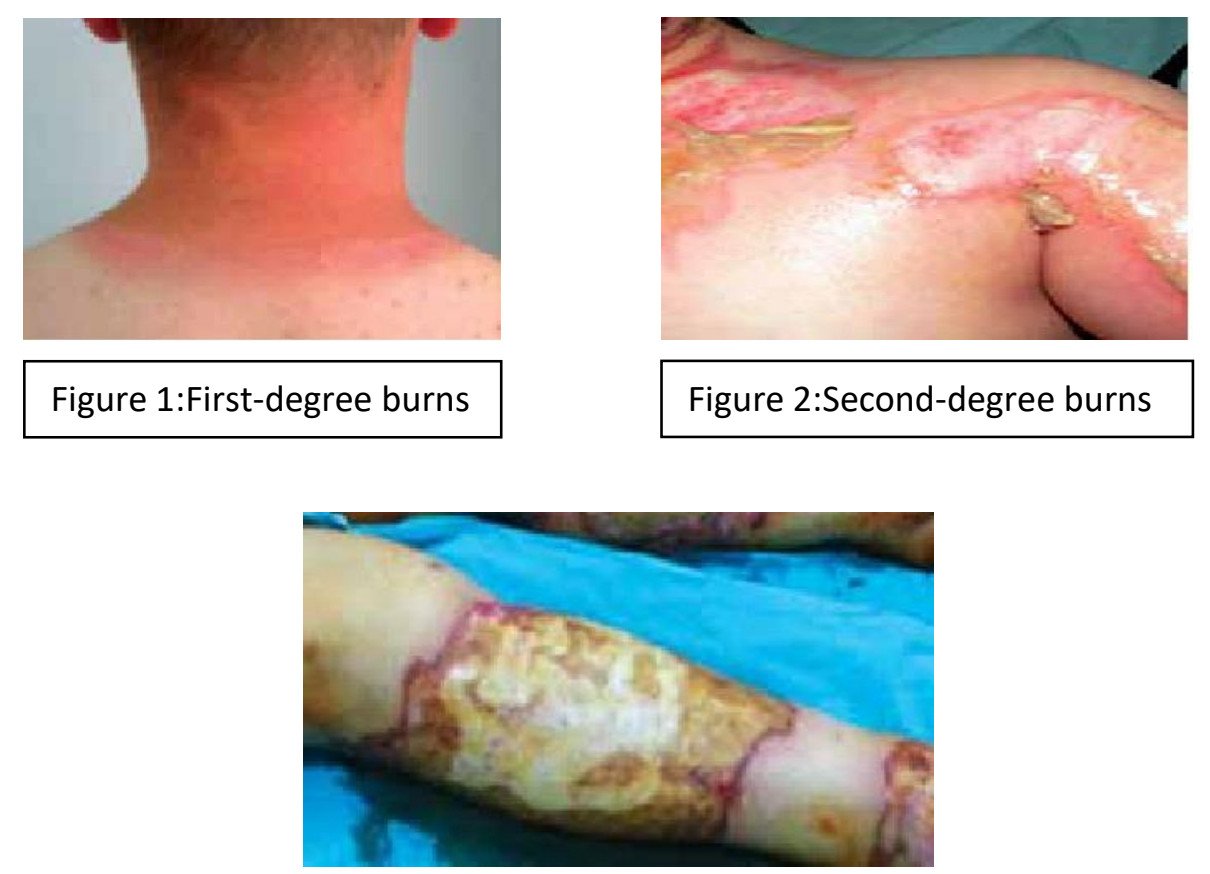

Figure 3: Third-degree burns

\section{Conclusion}

Burn patients should be evaluated in detail. Burns are divided into 4 stages according to the classification. First-degree burns affect only the epidermis, second-degree burns affect certain layers of the dermis, third-degree burns include all layers of the skin and fourth-degree burns include the muscles, tendons and bones. Second-degree superficial burns are red, pink, wet and painful $(1,2)$. There is decreased sensation but capillary filling is present. These burns are expected to improve in 10-14 days. Deep dermal burns are cherry red in color and have a dry surface. There is no sensation or capillary filling. They usually improve within 3-8 weeks if no infection develops. There is a tendency to scar formation due to the long inflammatory process during recovery $(1,2,3,4)$. Our cases included patients with first-, second- 
, and third-degree burns. Most of our patients were children, similar to the literature $(3,4)$. The burn area is usually calculated using the rule of nines in burn patients (4).It is appropriate to start the treatment by calculating the percentage of the burn area. We also started the treatment after calculating the burn area in our study. Sunscreen creams, aloe vera creams and moisturizing creams are used twice a day in addition to antihistamines in the treatment of first-degree burns. It is important to provide analgesia. The patient can be hospitalized and treated as an inpatient to relieve the pain and provide the necessary hydration in large first-degree burns. Surgery is not required in the treatment of second-degree superficial dermal burns and these can be treated with the appropriate creams and dressings (2). Paraffinimpregnated fabrics will decrease the pain during dressing changes by avoiding adherence to the wound. Polyurethane film sheets can also be used in cosmetically visible areas. If these cannot be provided, gauzes impregnated with paraffin or oily ointments (e.g., $0.2 \%$ Nitrofurazone ointment) can be used. Small blisters can be left in place. Large blisters should be drained or removed and followed-up with dressings. Antibiotic creams containing silver sulfadiazine, mupirocin and nitrofurazone can be applied directly to second-degree deep dermal burns. Such burns improve but result in a significant scar without surgical intervention. Surgical intervention is recommended for better healing with a smaller scar $(2,3$, $5,6,7,8)$. It is best to apply the proper dressing and transfer the patient to a burn unit/center without delay. Third-degree burns are treated with serial excision of the scars and skin grafting. Sunscreen creams and analgesics were prescribed for our patients with first-degree burns. The patient was called for weekly follow-up. The duration until recovery in these patients was 7 to 10 days. Our second-degree burn patients were hospitalized and treated by with antibiotic creams containing sulfadiazine and applying dressings under sedation. The mean duration of improvement varied between 3 and 6 weeks. Our patients with third-degree burns underwent debridement in the operating room. They were then treated by removing partial thickness skin grafts from intact skin tissue with a dermatome and placing them on the debrided areas. Daily checks were performed and dressings with antibiotic creams applied under sedation. Although trying to prevent the development of functional disability in the joint areas is mandatory in the early stages, it requires a strong and continuous effort. It is quite difficult to differentiate the care in the acute stage from burn rehabilitation because pain, limitation of movement due to inflammation and the accompanying scar, and the long-term adverse musculoskeletal dysfunction are affected by events that mostly occur in the early period $(8,9)$. Although pain management is a major challenge for clinicians, proper pain control is the basis for effective burn care, from the initial injury to long-term recovery (9). Studies should therefore be continued with a multidisciplinary approach to understand the pathophysiology of inflammation in burns in order to develop preventive interventions and treatments affecting the functional result. Continuing the hypermetabolic treatment after a burn has resulted in significant improvements in the growth and development of children in particular. $(8,9,10,11)$. Suggested issues to study to improve the functional results following burns include the medical, surgical approaches to prevent heterotopic ossifications due to the scars and immobilization, scar modulation with laser treatment, and skin replacements. Psychosocial problems that develop after burn injury may become permanent over time. Psychosocial interventions may be required for many years after burn injuries. Psychological and physical rehabilitation should be started and managed in an interdisciplinary manner during and after the burn period $(6,7,8,9,10,11)$. Physiotherapy and psychiatry consultations were requested for the patients in our study. Some of our patients were started psychiatric treatment. The required exercises to prevent contracture development were implemented by the psychotherapist. Pain control was provided by the anesthesiologists.

In conclusion, the proper intervention can prevent deepening of the burn, infection and fluid loss in the early period but a multidisciplinary approach is required to arrange the appropriate treatment for the burn patient.

Conflict of Interest $\quad$ : No conflict of interest was declared by the authors.

Financial Disclosure $\quad$ : No

\section{$\underline{\text { References: }}$}

1-Therapy of burns. Daigeler A., Kapalschinski N., Lehnhardt M. Chirurg, 2015 Apr;86(4):389-401. doi: 10.1007/s00104-014-2919-3.[Article in German].

2-Antimicrobial treatment in burn injury patients. Trupkovic T ., Gille J., Fischer H., Kleinschmidt S. Anaesthesist. 2012 Mar;61(3):249-51, 254-6, 258. doi: 10.1007/s00101-012-1994-4. [Article in German]. 
3-Keloid and hypertrophic scar treatment modalities. An update. Branski LK ., Rennekampff HO, Vogt PM. Chirurg. 2012 Sep;83(9):831-4, quiz 845-6. doi: 10.1007/s00104-011-2243-0. [Article in German]

4-Intensive Care Management of Pediatric Burn Patients. Ayse Ebru Sakallığlu Abalı. J Turk Soc Intens Care 2011;9(1):

5-Burn injury: Challenges and advances in burn wound healing, infection, pain and scarring. Wang Y., Beekman J., Hew J ., Jackson S ., Issler-Fisher AC ., Parungao R., Lajevardi SS ., Li Z., Maitz PKM . Adv Drug Deliv Rev. 2018 Jan 1;123:3-17. doi: 10.1016/j.addr.2017.09.018. Epub 2017 Sep 20

6-Porcine models of cutaneous wound healing. Seaton M ., Hocking A ., Gibran NS . ILAR J. 2015;56(1):127-38. doi: 10.1093/ilar/ilv016.

7-Important developments in burn care. Zuo KJ ., Medina A., Tredget EE. Plast Reconstr Surg. 2017 Jan;139(1):120e-138e. doi: 10.1097/PRS.0000000000002908.

8-Functional Outcomes Following Burn Injury. Colleen M. Ryan., MD, Ingrid Parry., MS, PT., Reginald Richard, MS, PT. Journal of Burn Care \& Research, Volume 38, Issue 3, May-June 2017, Pages 614-e617

9-Sedation and Pain Management in Burn Patients Cornelia Griggs, Jeremy Goverman, Edward Bittner, and Benjamin Levi. Clin Plast Surg. 2017 Jul; 44(3): 535-540. Published online 2017 Apr 18. doi: 10.1016/j.cps.2017.02.026

10-Update on metabolism and nutrition therapy in critically ill burn patients. Moreira E, Burghi G, Manzanares W. Med Intensiva. 2018 Jul;42(5):306-316. doi: 10.1016/j.medin.2017.07.007. Epub 2017 Sep 23. Review. English, Spanish.

11-Psychosocial Distress at Different Time Intervals after Burn Injury. Berg L ., Meyer S ., Ipaktchi R ., Vogt PM ., Müller A ., de Zwaan M . Psychother Psychosom Med Psychol. 2017 Jun;67(6):231-239. doi: 10.1055/s-0042-111006. Epub 2016 Sep 29. [Article in German; Abstract available in German from the publisher] 